\title{
DNS of compressible turbulent boundary layer around a sharp cone
}

\author{
LI XinLiang ${ }^{\dagger}$, FU DeXun \& MA YanWen
}

State Key Laboratory of Nonlinear Mechanics, Institute of Mechanics, Chinese Academy of Sciences, Beijing 100080, China

Direct numerical simulation of the turbulent boundary layer over a sharp cone with $20^{\circ}$ cone angle (or $10^{\circ}$ half-cone angle) is performed by using the mixed seventhorder up-wind biased finite difference scheme and sixth-order central difference scheme. The free stream Mach number is $\mathbf{0 . 7}$ and free stream unit Reynolds number is 250000/inch. The characteristics of transition and turbulence of the sharp cone boundary layer are compared with those of the flat plate boundary layer. Statistics of fully developed turbulent flow agree well with the experimental and theoretical data for the turbulent flat-plate boundary layer flow. The near wall streak-like structure is shown and the average space between streaks (normalized by the local wall unit) keeps approximately invariable at different streamwise locations. The turbulent energy equation in the cylindrical coordinate is given and turbulent energy budget is studied. The computed results show that the effect of circumferential curvature on turbulence characteristics is not obvious.

direct numerical simulation, boundary layer transition, sharp-cone, turbulent kinetic energy budget

\section{Introduction}

The boundary layer flows over flying vehicles determine the layouts of aerodynamic force and aerodynamic heating. And correct prediction of laminar-turbulent transition in boundary layers is very important for the aerodynamic design and control of flying vehicles. As a typical leading shape of high-speed flying vehicles, the transition flow over a sharp or blunt cone is very interesting in aeronautics and astronautics. Because of practical needs, there are lots of flight and wind tunnel experiments for boundary-layer transition (see ref. [1] for an overview), and the measurements of transition locations are dominant contents of these experiments. However, the agreements between different experiments are not so good, especially, there are distinct differences between flight experiments and wind tunnel experiments. Sharp cones are considered as typical shapes of

Received January 15, 2007; accepted May 22, 2007 doi: $10.1007 / \mathrm{s} 11433-008-0007-8$

${ }^{\dagger}$ Corresponding author (email: lixl@lnm.imech.ac.cn)

Supported by the National Natural Science Foundation of China (Grant Nos. 10632050 and 10502052) and Informatization Construction of Knowledge Innovation Projects of the Chinese Academy of Sciences (Grant No. INF105-SCE) 
flying vehicles, and NACA/NASA performed lots of experiments about the turbulent flow and transitions of boundary around sharp cones ${ }^{[2-4]}$.

The most used transition prediction method is $e^{N}$ method, but this method has a limit that the initial amplitude of disturbance is not considered, as addressed in ref. [5]. However, the transition process depends strongly on the initial disturbance. The characteristics of initial disturbance, including the amplitudes, frequencies, wave numbers and others, can be better understood through the study of receptivity ${ }^{[5]}$. Ma et al. ${ }^{[5]}$ and Zhong et al. ${ }^{[6]}$ studied the receptivity of supersonic boundary layers to free stream disturbance. According to Ma and Zhong's study, there is a family of stable wave modes in the supersonic boundary layer, in addition to the well-known unstable Mack modes. And these stable wave modes play important roles in the receptivity process through resonant interactions with both fast sound wave and unstable Mack modes. Besides free-stream disturbance, surface roughness is also disturbance source for transition. Since the disturbance amplitude of high-altitude atmosphere is very small, wall roughness may play an important role in the boundary layer transition of high-altitude flying or re-entry. Zhong ${ }^{[7]}$ studied the effects of surface roughness on the receptivity of hypersonic blunt-cone boundary layer by numerical simulation, and found that the rough element generates streamwise vortices inside the boundary layer. However, the research work of the surface roughness receptivity is very little now and needs to be paid more attention to.

Due to developments of computational techniques and powerful computers, direct numerical simulation (DNS) becomes a more and more important tool in studying turbulent flow and transitions. Because of complexity, DNS of compressible turbulent boundary layer flows did not start until recent years. Guarini et al. ${ }^{[8]}$ performed DNS of a flat-plate boundary layer with free stream Mach number 2.5; Maeder et al. ${ }^{[9]}$ performed the same DNS with free stream Mach number 3, 4.5 and 6, and also the compressibility effects were studied. Both Guarini and Maeder used the temporal evolving mode. With this kind of modes it is difficult to study the development of the spatial boundary layer transition. Rai et al. ${ }^{[10]}$ performed DNS for the spatially evolving flat-plate boundary at free stream Mach number 2.25 by using the 5 th order upwind biased finite difference scheme for convection terms, and the 4th order center order scheme for viscous terms. Pirozzoli et al. ${ }^{[11]}$ performed the same DNS case later by using the 7 th order WENO scheme for convection terms and the 4th order Pade scheme for viscous terms, and the effects of numerical scheme's resolution on the mean velocity were studied. Compared to the temporally evolving one, the spatially evolving DNS need not "slow growth" or "extended temporal" assumptions and thus minimizes any uncertainty or artificial assumption ${ }^{[11]}$. Gao et al. ${ }^{[12]}$ also performed the DNS of compressible boundary layer at $M a=2.25$, and found that the spanwise asymmetry of the disturbance is very important to induce the bypass transition. Luo et al. ${ }^{[13]}$ performed numerical study on the transition of channel flow, and found that the change of mean velocity profile plays an important role in the transition. $\mathrm{Li}$ et al. ${ }^{[14,15]}$ performed direct numerical simulations of spatially evolving boundary layers over a blunt wedge and a flat plate with the free stream Mach number $M a_{\infty}=6$, and the mean pressure gradient and compressibility effects on the vortex structure were studied.

Until now, there is still no report on DNS of the compressible turbulent boundary layer over sharp cones in which the aerodynamicists are much more interested. In this paper, DNS of compressible turbulent boundary layer around a $20^{\circ}$ cone angle (or $10^{\circ}$ half-cone angle) sharp cone is performed. The wall blowing and suction perturbations are used to induce the laminar to turbulent transition, and in order to speed up the transition process, the chosen amplitude of perturbations is 
much stronger than that for flat-plate boundary layers ${ }^{[11]}$. The characteristics of transition and turbulence of the sharp cone boundary layer are compared with those of the flat plate boundary layer. The computed results show that although the transition location moves upstream and the transition region reduces, the basic characteristics and statistics of fully developed turbulence agree well with the flat-plate boundary layer. The near wall streak-like structure is shown and the space between streaks (normalized by wall unit) keeps approximately invariable at the different streamwise location. The turbulent energy equation in the cylindrical coordinate is given and turbulent energy budget is studied. Deduction of the turbulent energy equation in the cylindrical coordinate can be seen in the appendix.

\section{Numerical simulation}

Consider the flow field around a $20^{\circ}$ cone angle (or $10^{\circ}$ half-cone angle) sharp cone with zero angle of attack (see Figure 1).

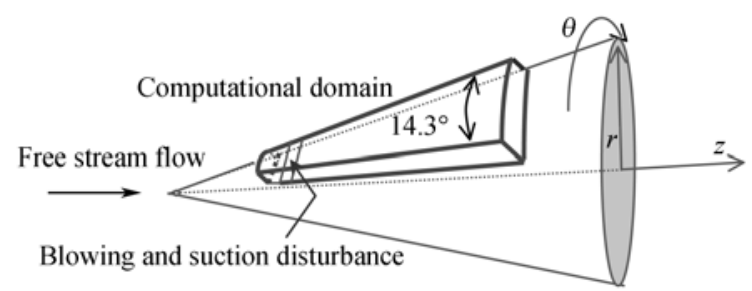

Figure 1 Sketch of the sharp cone and three-dimensional computational domain.

In the first step, a two-dimensional (axial-symmetrical) steady state flow is computed to provide initial and boundary conditions for three-dimensional computation. To avoid numerical singularity, a small round leading edge is used instead of the sharp leading edge, and the radius of leading edge is chosen consulting the real leading edge radius in the experiments ${ }^{[3,4]}$, i.e. the cone is not absolutely "sharp" but blunt.

For actual hypersonic vehicles, the bluntness of the leading edge has significant effects on the transition location ${ }^{[16]}$. The reason may be related to the entropy layer and the leading-edge receptivity. For a supersonic or hypersonic boundary layer, the leading edge bluntness affects significantly the boundary-layer outer flow through the entropy layer generated by the bow-shock. And the leading edge bluntness also affects the initial disturbance through the leading-edge receptivity. The authors will study the leading-edge-effects in the future. In this research, since the free stream flow is subsonic, there is no bow-shock over the boundary layer and no entropy layer generated by the bow-shock. Additionally, wall blowing and suction disturbance is imposed, which is much stronger than the disturbance from the leading edge. So, leading-edge-effects are not significant in this research, and thus are not considered.

Computational parameters and grid parameters are shown in Table 1. The free stream Mach number is $M a_{\infty}=0.7$, the free stream Reynolds number (using an inch as length unit) is $R e_{\infty}=$ 250000, the momentum thickness Reynolds number is $R e_{\theta}=877$ (using the momentum thickness at $z=2.5$ ), and the isothermal wall's temperature is $T_{\mathrm{w}}=1.098$.

The sketch of the computational domain and meshes in axial-section is shown in Figures 1 and 2. The computational domain for three-dimensional simulation is $1.22 \leqslant z \leqslant 4.14, \eta_{\max }=0.225,0 \leqslant$ 
Table 1 Computational parameters and mesh parameters

\begin{tabular}{cccccc}
\hline$M a_{\infty}$ & $R e_{\infty}$ & $T_{\mathrm{w}}$ & $R e_{\theta}$ & $N_{\xi} \times N_{\eta} \times N_{\varsigma}$ & $\Delta \xi^{+} \times \Delta \eta_{\mathrm{w}}^{+} \times \Delta \zeta^{+}$ \\
\hline 0.7 & 250000 & 1.098 & 877 & $1050 \times 100 \times 200$ & $19 \times 1.2 \times 6.8$ \\
\hline
\end{tabular}

$\theta \leqslant 0.25$, where $\eta$ denotes the vertical distance to the wall (see Figure 2). To save computational resource, the computation is carried out in the circumferential direction that only occupies 0.25 radium. $\left(\approx 14.3^{\circ}\right)$. The circumferential (spanwise) two-point correlations show that the spanwise space of the computational domain is large enough. The number of computational mesh is $1050 \times 100 \times 200 \quad(z, \theta, r$-direction, respectively). The progressively coarsened grid at $z>3.1$ is used as a buffer region to inhibit the reflection of numerical disturbance at the outlet ${ }^{[11]}$. Streamwise mesh spacing is approximately uniform in the main computation region, and $\Delta \xi^{+} \approx 19$; the wall normal first mesh spacing is $\Delta \eta_{\mathrm{w}}^{+}=1.2$; and the spanwise (circumferential) mesh spacing at $z=2.5$ is $\Delta \zeta^{+}=6.8$.

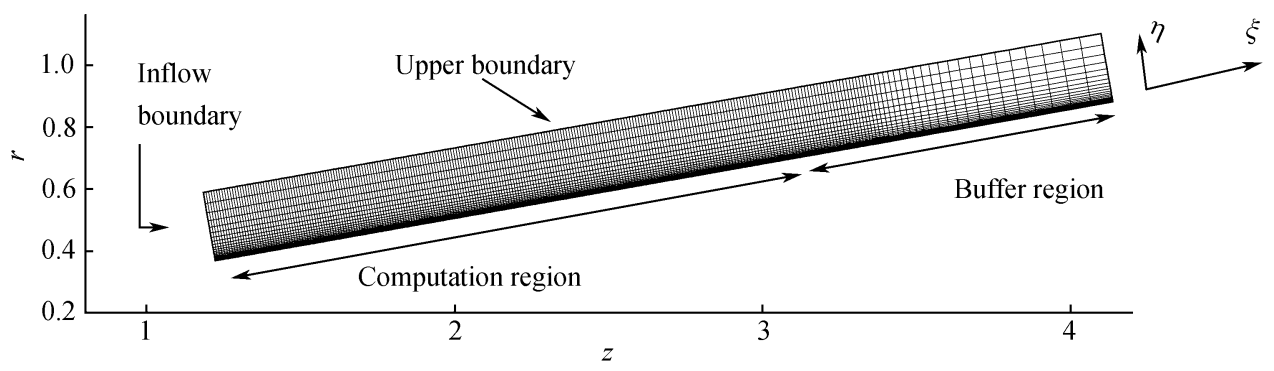

Figure 2 Sketch of computational meshes in the axial-section (1/4 mesh of each direction is visualized).

The compressible Navier-Stokes equations at cylindrical coordinates $(r, \theta, z)$ are used and $\rho$, $u_{r}, u_{\theta}, u_{z}, T$ are used as the fundamental variables. Jacobian transform $r=r(\xi, \eta), z=z(\xi, \eta)$, $\theta=\theta$ is used to transform the original equations to the rotational coordinates $(\xi, \eta, \theta)$. The reason of using Jacobian transformed cylindrical coordinate equations is that all the variables $\left(\rho, u_{r}, u_{\theta}, u_{z}, T\right)$ are periodical in $\theta$-direction. If Jacobian transformed Cartesian equations are used, the $\theta$-direction boundary conditions of $u_{x}, u_{y}$ will be rather complicated.

At the inflow boundary and upper boundary, time-independent conditions obtained from twodimensional (axial-symmetry) simulation are imposed. In this paper, the computational domain at the wall normal direction is fairly large (6-7 times the boundary layer thickness), and the numerical disturbances reflected from the upper boundary is very weak (see Figure 12). At the outflow boundary, non-reflecting boundary condition is used, and no-slip condition is used at the wall boundary with assumption $\partial p / \partial \eta=0$.

High order accurate finite difference method is used in our computation. The convection terms are approximated by using the 7th order upwind biased difference scheme after flux splitting, the viscous terms are approximated by using the 6th order central difference scheme, and the 3rd order TVD type Runge-Kutta method is used for time stepping.

The blow and suction disturbance $e^{[10,11]}$ is imposed at the wall of the interval $1.5=z_{a} \leqslant z \leqslant z_{b}=$ 1.8 , and the form of the disturbance is shown as follows ${ }^{[10,11]}$ : 


$$
\begin{aligned}
& v_{\mathrm{bs}}=A u_{\infty} f(z) g(\theta) h(t), \\
& f(x)=4 \sin \alpha(1-\cos \alpha) /(27)^{1 / 2}, \\
& g(\theta)=\sum_{l=0}^{l_{\max }} Z_{l} \sin \left(2 \pi l\left(\theta / \theta_{\max }+\phi_{l}\right)\right), \\
& h(t)=\sum_{m=0}^{m_{\max }} T_{m} \sin \left(2 \pi m\left(\beta t+\phi_{m}\right)\right),
\end{aligned}
$$

where

$$
\begin{aligned}
& \alpha=2 \pi\left(z-z_{a}\right) /\left(z_{b}-z_{a}\right), \\
& \sum_{l=0}^{l_{\max }} Z_{l}=1, \quad Z_{l}=1.25 Z_{l+1}, \\
& \sum_{m=0}^{m_{\max }} T_{m}=1, \quad T_{m}=1.25 T_{m+1},
\end{aligned}
$$

where $l_{\max }=10, m_{\max }=5,\left[z_{a}, z_{b}\right]$ is an interval in the streamwise direction in which the disturbance is given; $\theta_{\max }=0.25$ is the span of circumferential computational domain; $\phi_{l}, \phi_{m}$ are random numbers between 0 and $1 ; A=0.2$ is amputate of perturbation, and $\beta=2.0$ is base frequency.

With the support of Super Computing Center of Chinese Academy of Sciences (SCCAS), we have enclosed all codes to a new software named "Hoam-OpenCFD" (OpenCFD). OpenCFD is a high accuracy CFD software developed by us, and the core of the software is a high accuracy finite difference solver for the compressible Navier-Stokes equations. This software supports many coordinates, such as Cartesian coordinate, cylindrical coordinate, spherical coordinate, curvilinear coordinate and rotational coordinate. Many popular finite difference schemes are included in this software, such as central difference schemes, upwind difference schemes, compact difference schemes, WENO schemes, and so on. Some schemes developed by us (such as upwind compact schemes ${ }^{[17,18]}$ and GVC schemes ${ }^{[19]}$ ) are also included in this software. The software is coded by using MPI-Fortran90 and has fairly high parallel performance for supercomputers and PC Clusters.

\section{Statistics and instantaneous structure}

Computation is performed on the super computer Lenovo Deep-comp 6800 (the CPUs are Itanium 2 at $1.3 \mathrm{GHz}$ ) of SCCAS, and 20 nodes (we choose $2 \mathrm{CPUs} /$ node) are used for the computation. Mean performance is $8.3 \mathrm{~s} /$ time-step. The turbulence becomes stationary when $t=8.0$, then we saved the flow data at $t=8.7-12.1$ (total 35 data files), and the statistics is based on these data files. Some turbulence statistics are presented and compared with the experimental and theoretical results for the flat-plate boundary layer flow.

Figure 3 shows the skin friction on the wall in the streamwise direction, and the figure shows that skin friction increases very fast after the disturbance region. The skin friction is defined as $C_{\mathrm{f}}=\tau_{\mathrm{w}} / \frac{1}{2} \rho_{\infty} u_{\infty}^{2}$, where $\tau_{\mathrm{w}}=\left.\mu_{\mathrm{w}} \frac{\partial \bar{u}}{\partial y}\right|_{\mathrm{w}}$. According to ref. [1], the transition location in flight experiments is about $R e_{x} \approx 10^{6}-10^{7}$, and the transition location in our study is $R e_{x} \approx 0.5 \times 10^{6}$ 
(based on the distance to the cone tip). Because the perturbations in this study are much stronger than that in flight experiments, the transition is more fast and early.

Figure 4 shows the mean streamwise velocities at $z=1.43,1.85,2.06,2.28$ and 2.50. From this figure we can see the profiles become chubbier as $z$ increases. Figure 5 shows the mean stream velocity $\bar{u}$ and mean Van Driest velocity $\bar{u}_{\mathrm{vd}}$ profile normalized by the wall-shear velocity at $z=$ 2.5. Because the Mach number is not high, especially, the wall temperature is close to that of the free stream flow, the difference between $\bar{u}$ and $\bar{u}_{\mathrm{vd}}$ is very small. The log law for the flat-plate boundary layer expressed as $u^{+}=1 / \kappa \ln y^{+}+5.5$ (where $\kappa=0.41$ ) is also shown in this figure. From this figure we can see that the computational result agrees with the log law very well.

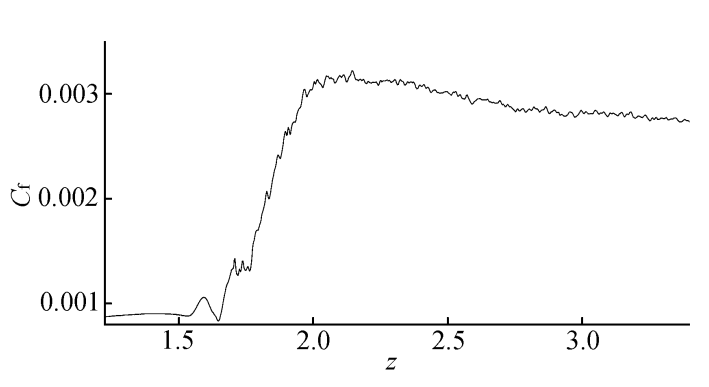

Figure 3 Skin friction distribution along $z$-direction.

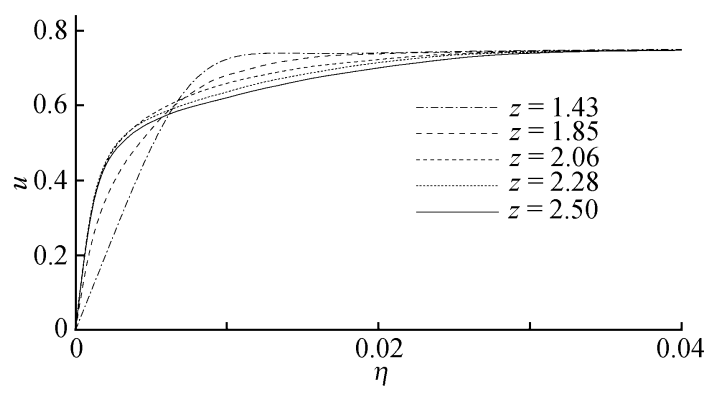

Figure 4 Mean streamwise velocities at different $z$-location.

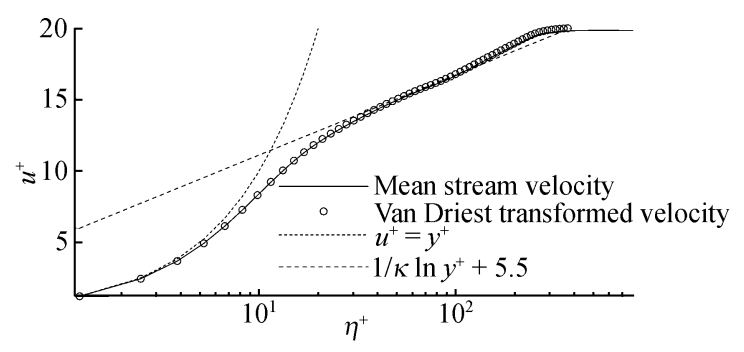

Figure 5 Profile of mean streamwise velocity at $z=2.5$.

Figure 6 shows the circumferential (spanwise) two-point correlations at the different vertical position for $z=2.25$. Form this figure we can see that the correlations decay toward zero near the tails to the distribution, which shows that the spanwise span of the computational domain is enough.

Figure 7 shows the normalized turbulence intensities $u_{\mathrm{rms}}^{\prime} / \bar{u}, v_{\mathrm{rms}}^{\prime} / \bar{u}, w_{\mathrm{rms}}^{\prime} / \bar{u}$ at $z=2.5$ as a function of the vertical distance to the wall. We can see that in the near-wall region $u_{\mathrm{rms}}^{\prime}>w_{\mathrm{rms}}^{\prime}>$ $v_{\text {rms }}^{\prime}$, which means that the turbulence is strongly anisotropic near the wall. The symbols in this figure denote the experimental data for the incompressible flat-plate boundary layer ${ }^{[20]}$, and we can see that the computational data agrees well with these experimental data.

Figure 8 shows the normalized Reynolds shear stress $-\overline{u^{\prime} v^{\prime}} / u_{\tau}^{2}$ at $z=2.5$, where the symbols denote the experimental data for the incompressible flat-plate boundary layer ${ }^{[20]}$. We can see that the computational data matches the experiment data very well.

The symbols in Figures 5, 7 and 8 denote the experimental or theoretical data for the flat-plate 

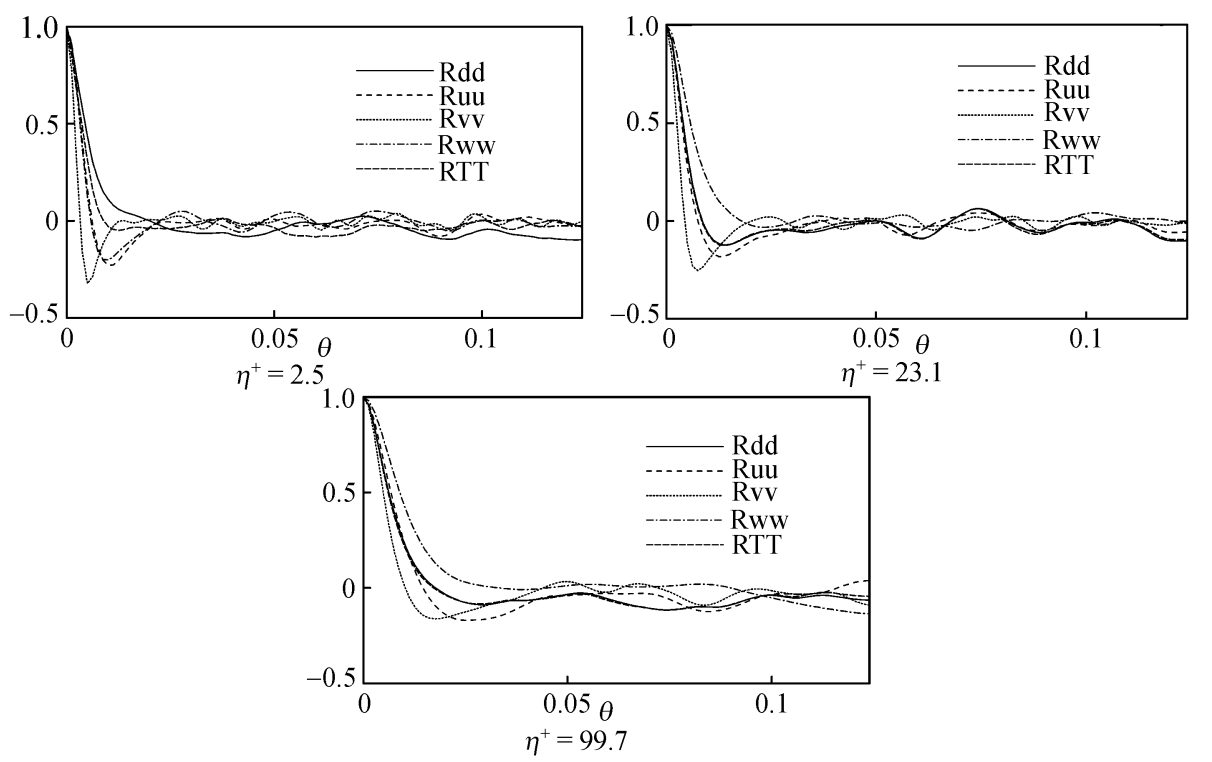

Figure 6 Two-point correlations in the circumferential direction.

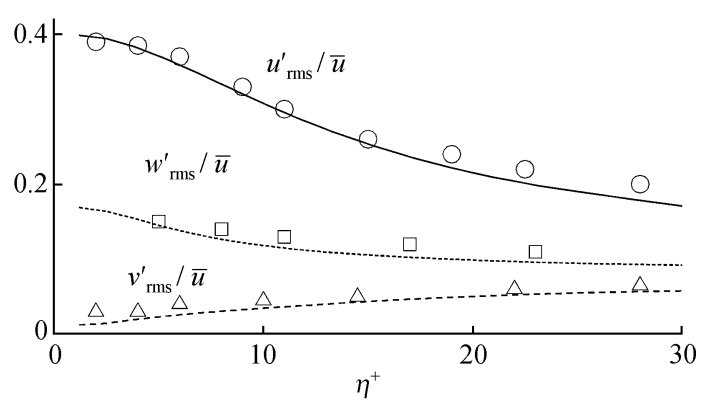

Figure 7 The normalized turbulent intensities at $z=2.5$. Lines denote computational data and symbols denote experimental data.

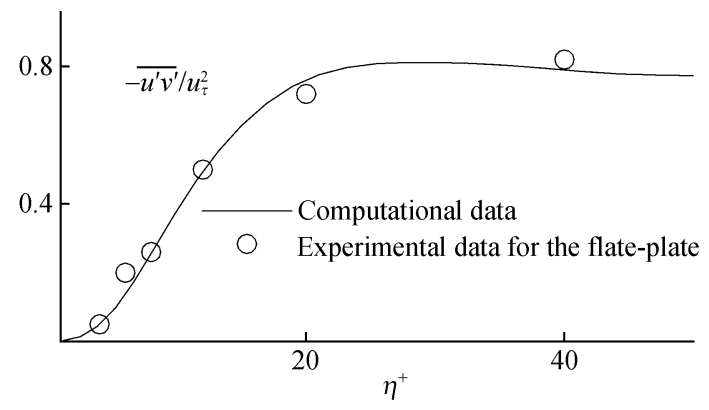

Figure 8 Normalized Reynolds shear stress at $z=2.5$.

boundary layer. The agreements between these data and the turbulent statistics for sharp-cone boundary layer turbulence show that circumferential curvature has little effect on the near wall turbulence statistics.

Streamwise velocity contours on the curved surface at $\eta^{+}=2.5,23.1$ and 99.7 are shown in Figure 9. From this figure we can see that there are clearly streamwise streaky structures near the wall and the streaky structures become indistinct at the far-form-wall district. We can also see the transition skips the T-S and the second instability and the transition process is a bypass transition process.

Figure 10 shows the circumferential two-point collections at $z=2.28,2.50,2.73,2.97 . \eta=$ 0.00027 is for all these cases $\left(\eta^{+}=2.5\right.$, normalized by the wall unit of $\left.z=2.5\right)$. The horizontal coordinate $\zeta^{+}=\zeta / l^{*}$ is the circumferential distance normalized by the local wall unit $l^{*}=$ $\bar{\mu}_{\mathrm{w}} /\left(\bar{\rho}_{\mathrm{w}} \sqrt{\tau_{\mathrm{w}} / \bar{\rho}_{\mathrm{w}}}\right)$. From this figure we can see that the negative maximum value is near $\varsigma^{+}=60$, which means that the mean space between high-speed-streak and low-speed-streak is $\varsigma^{+} \approx 60$, 


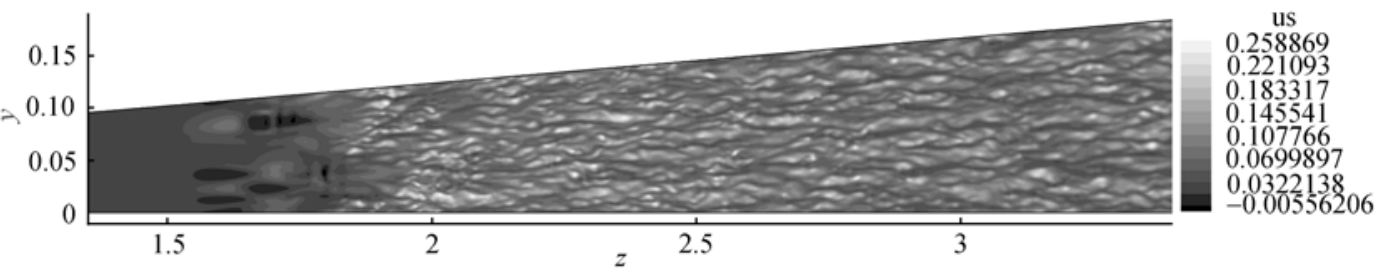

(a) $\eta^{+}=2.5$

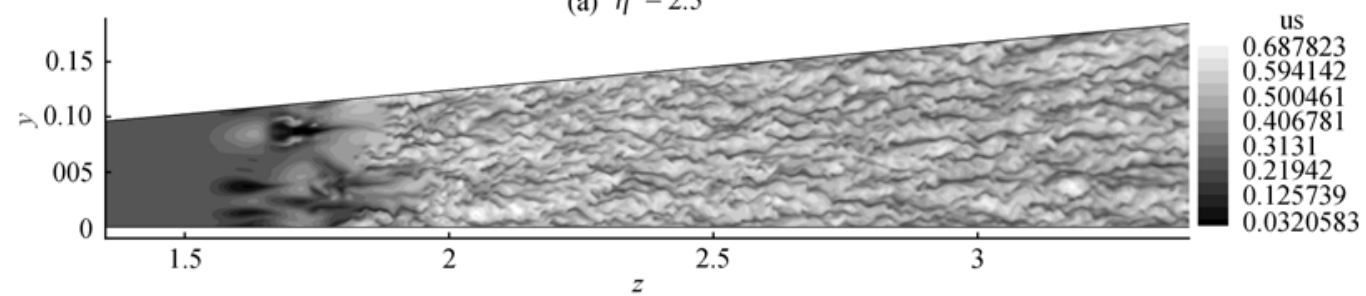

(b) $\eta^{+}=23.1$

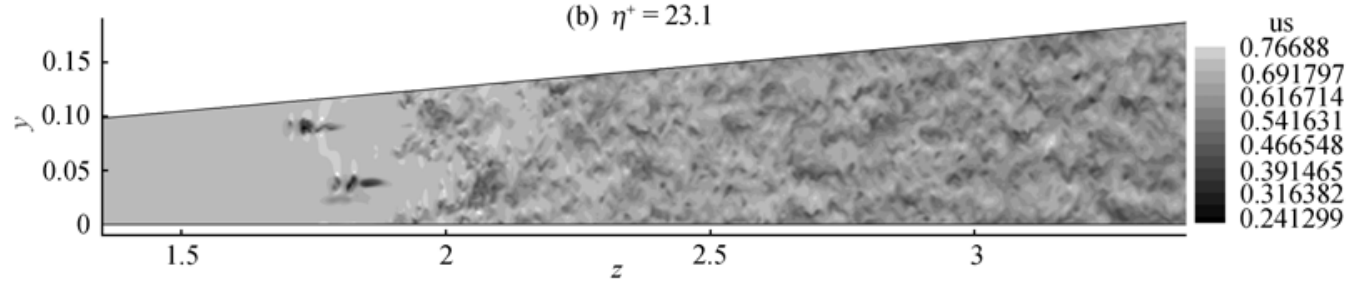

(c) $\eta^{+}=99.7$

Figure 9 Contours of streamwise velocity at difference curved faces (projected to $y$-z plane).

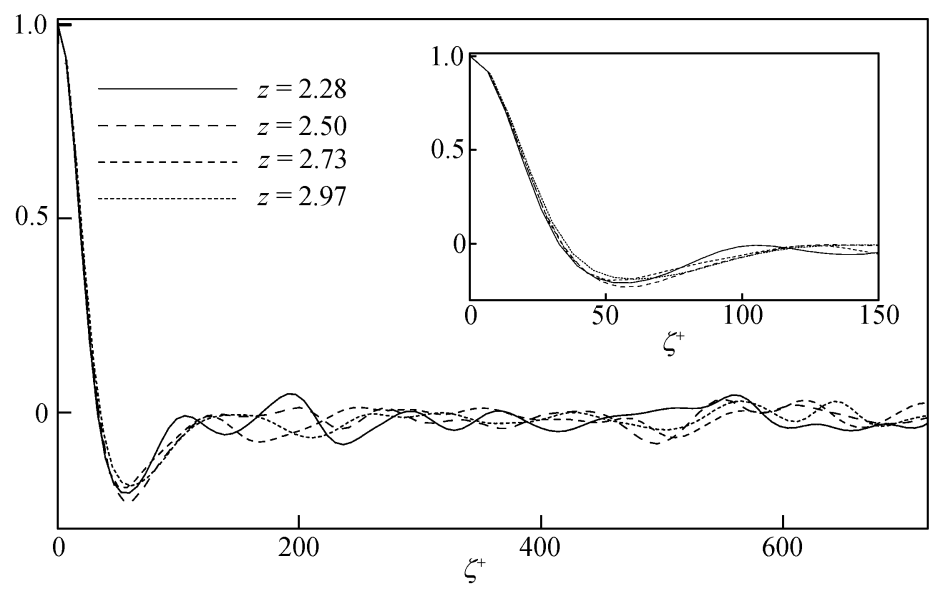

Figure 10 Circumferential two-point collections at different $z$-locations.

and so the mean space between high-speed-streaks (or low-speed streaks) is $\varsigma^{+} \approx 120$. We can see that the space between streaks does not enlarge along $z$-direction, and from the results we can infer that the number of streaks increases along $z$-direction.

Figures $11-13$ show the instantaneous density field at the wall-normal section, axial section, and wall-parallel section, respectively, which show highly intermittent characteristics of outer layer. From Figure 12 we can also see that the numerical disturbance near the upper boundary is negligible. 


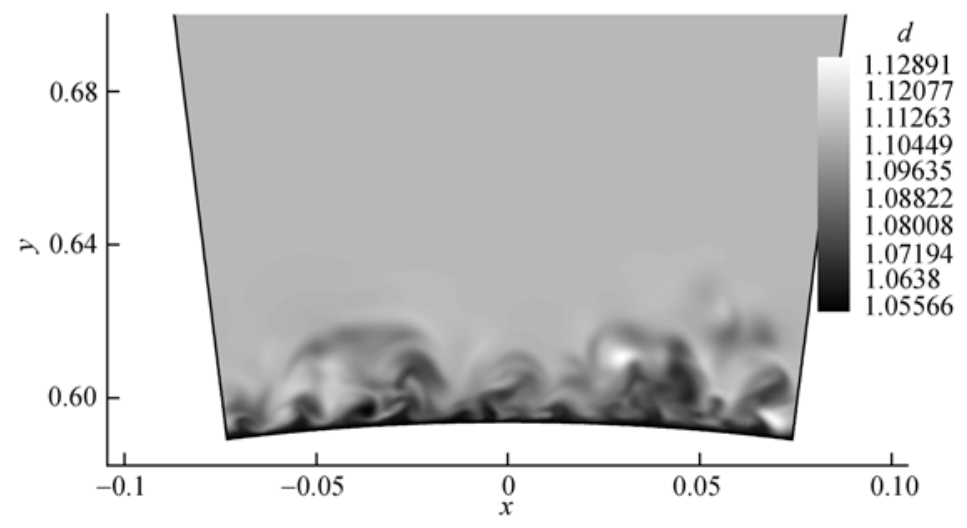

Figure 11 Instantaneous density field at the section normal to the wall $(z=2.5)$.
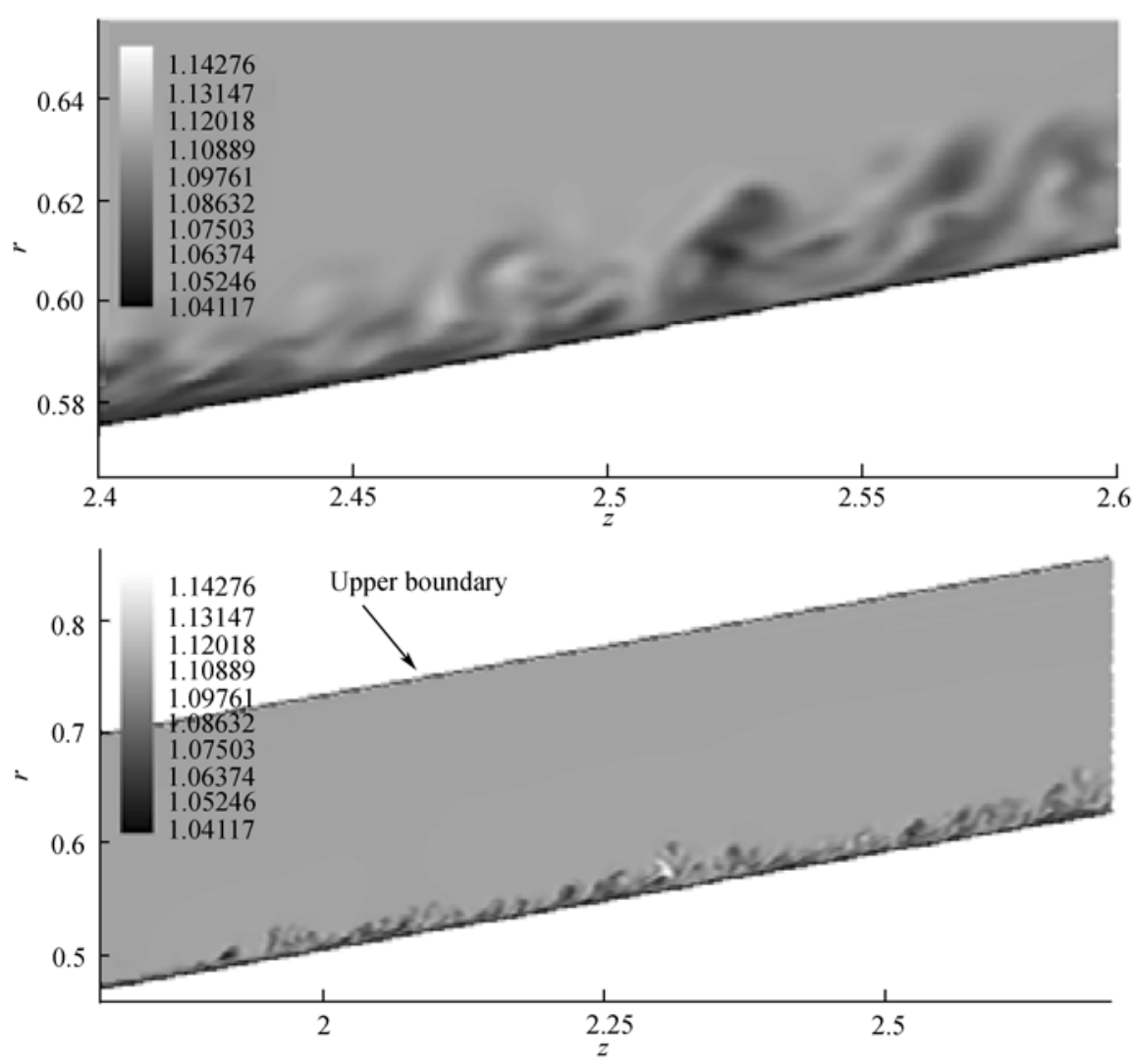

Figure 12 Instantaneous density field at the axial section.

The statistics and visualizations show that the near wall turbulent characteristics of the sharp cone boundary layer are very similar to those of a flat-plate boundary. This is because that the curvature radius of wall $R$ is much larger than the thickness of the boundary layer $\delta$ (in this case, $\delta / R<0.1$ ), so effects of circumferential curvature are not so significant.

Figure 14(a) shows the instantaneous isosurface of $Q^{+}=0.005$, where $Q^{+}=Q /\left(U_{\tau} / l^{*}\right)^{2}, U_{\tau}$ 


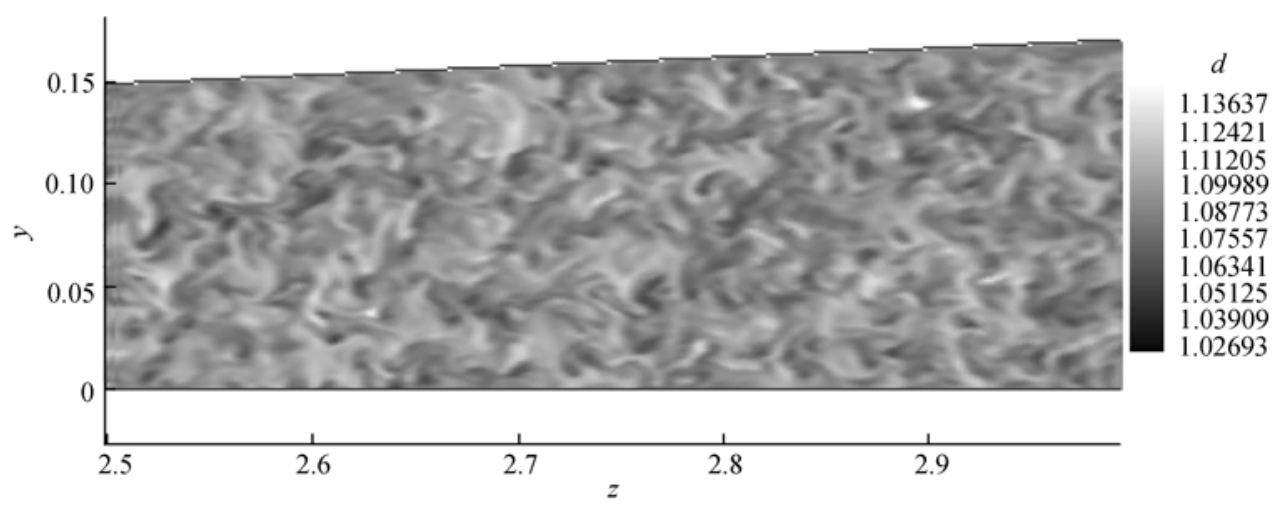

Figure 13 Instantaneous density field at the plane parallel to the wall at $\eta=0.011\left(\eta^{+}=99.7\right)$.

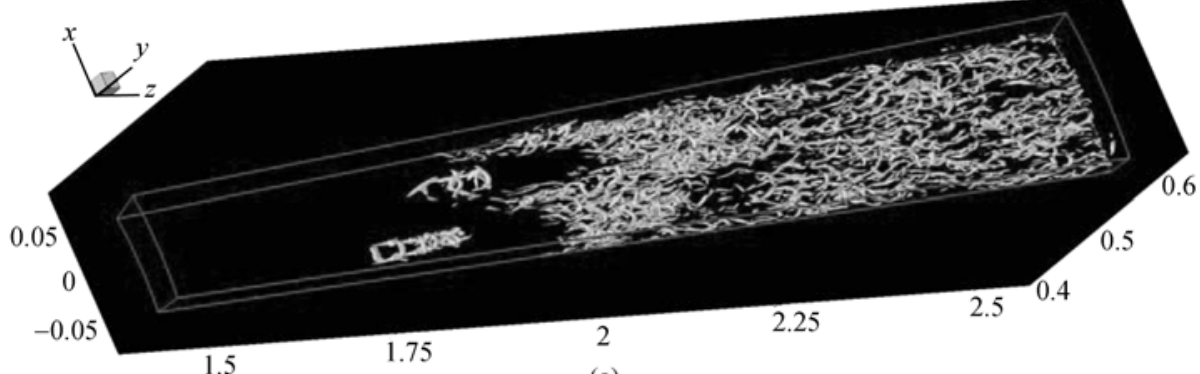

(a)

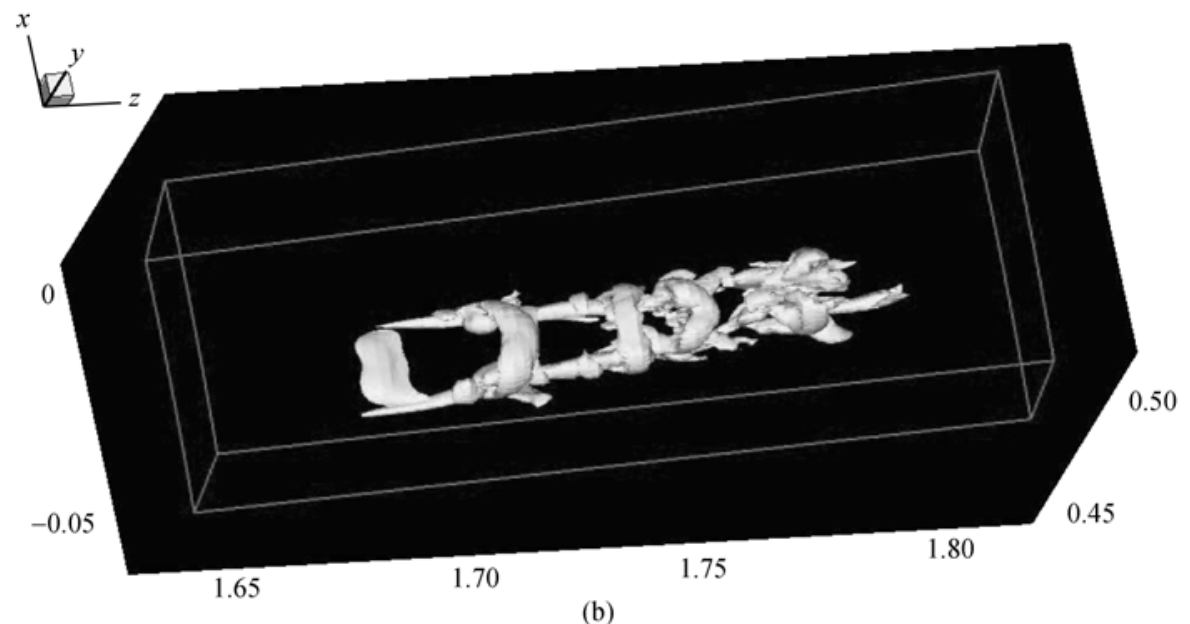

Figure 14 (a) Instantaneous isosurface of $Q^{+}=0.005$; (b) instantaneous isosurface of $Q^{+}=0.005$, the partially enlarged detail.

and $l^{*}$ are wall friction velocity and wall scale at $z=2.5$, respectively. $Q=I I\left(\frac{\partial u_{i}}{\partial x_{j}}\right)=$ $\frac{\partial u}{\partial x} \frac{\partial v}{\partial y}+\frac{\partial v}{\partial y} \frac{\partial w}{\partial z}+\frac{\partial u}{\partial x} \frac{\partial w}{\partial z}-\frac{\partial u}{\partial y} \frac{\partial v}{\partial x}-\frac{\partial v}{\partial z} \frac{\partial w}{\partial y}-\frac{\partial u}{\partial z} \frac{\partial w}{\partial x}$ is the second invariability of the velocity gradient tensor, and it is deemed that isosurface of $Q$ can describe the coherence structure well. From this figure, we can see that hairpin vortexes appear while transiting, and there is no two-dimension 
spanwise vortex and $\Lambda$-vortex before hairpin vortex. Figure $14(\mathrm{~b})$ is the partial enlarged detail of Figure 14(a), which shows hairpin vortex series, and one hairpin vortex can induce the downstream vortex.

\section{Turbulent kinetic energy budget}

The compressible turbulent kinetic energy equation for the cylindrical coordinate is shown as follows, and the deduction for this equation can be seen in the appendix.

$$
\frac{\partial}{\partial t}(\bar{\rho} K)=-C+P+T+\Pi_{t}+\Pi_{d}+D+M-\varepsilon
$$

where $C=\frac{\partial}{\partial x_{j}}\left(\tilde{u}_{j} \bar{\rho} K\right)+\frac{1}{r} \tilde{u}_{1} \bar{\rho} K$ is the convection term (in this and the following equations,

$$
\begin{aligned}
\frac{\partial}{\partial x_{1}}=\frac{\partial}{\partial r}, \frac{\partial}{\partial x_{2}}=\frac{1}{r} \frac{\partial}{\partial \theta}, \frac{\partial}{\partial x_{3}} & \left.=\frac{\partial}{\partial z}, u_{1}=u_{r}, u_{2}=u_{\theta}, u_{3}=u_{z}\right) \\
P & =-\overline{\rho u_{i}^{\prime \prime} u_{j}^{\prime \prime}} \frac{\partial \tilde{u}_{i}}{\partial x_{j}}+\frac{1}{r}\left(\tilde{u}_{2} \overline{\rho u_{1}^{\prime \prime} u_{2}^{\prime \prime}}-\tilde{u}_{1} \overline{\rho u_{2}^{\prime \prime} u_{2}^{\prime \prime}}\right)
\end{aligned}
$$

is the production term;

$$
T=-\left(\frac{\partial}{\partial x_{j}}\left(1 / 2 \overline{\rho u_{i}^{\prime \prime} u_{i}^{\prime \prime} u_{j}^{\prime \prime}}\right)+1 / 2 \frac{1}{r} \overline{\rho u_{i}^{\prime \prime} u_{i}^{\prime \prime} u_{1}^{\prime \prime}}\right)
$$

is the turbulent transport term;

$$
\Pi_{d}=\overline{p^{\prime}\left(\frac{\partial u_{i}^{\prime \prime}}{\partial x_{i}}+\frac{1}{r} u_{1}^{\prime \prime}\right)}
$$

is the pressure-dilatation term;

$$
\Pi_{t}=-\left(\frac{\partial}{\partial x_{j}} \overline{p^{\prime} u_{j}^{\prime \prime}}+\frac{1}{r} \overline{p^{\prime} u_{1}^{\prime \prime}}\right)
$$

is the pressure transport term;

$$
M=\bar{u}_{i}^{\prime \prime}\left(\frac{\partial \bar{\sigma}_{i j}}{\partial x_{j}}-\frac{\partial \bar{p}}{\partial x_{i}}\right)+\frac{1}{r}\left(\overline{u_{1}^{\prime \prime}}\left(\bar{\sigma}_{11}-\bar{\sigma}_{12}\right)+2 \overline{u_{2}^{\prime \prime}} \bar{\sigma}_{12}+\overline{u_{3}^{\prime \prime}} \bar{\sigma}_{13}\right)
$$

is the term associated with density fluctuations;

$$
D=\frac{\partial}{\partial x_{i}} \overline{u_{i}^{\prime \prime} \sigma_{i j}^{\prime}}+\frac{1}{r} \overline{u_{i}^{\prime \prime} \sigma_{i 1}^{\prime}}
$$

is the viscous diffusion term;

$$
\varepsilon=\overline{\sigma_{i j}^{\prime} \frac{\partial u_{i}^{\prime \prime}}{\partial x_{j}}}+\frac{1}{r} \overline{\sigma_{12}^{\prime} u_{2}^{\prime \prime}-\sigma_{12}^{\prime} u_{1}^{\prime \prime}}
$$

is the viscous dissipation term.

The comparison with the turbulent energy equation in Cartesian coordinate shows that there are some additional terms with $r$ in the denominator in the turbulent energy equation for the cylindrical coordinate, and we find that all these additional terms are very small in this DNS case.

We can see that the convection term $C$, turbulent transport term $T$, viscous diffusion term $D$ and 
pressure transport term $\Pi_{t}$ are all with the form of vector's divergence (The expression of vector's divergence in the cylindrical coordinate is $\left.\operatorname{div}(\boldsymbol{u})=\frac{\partial u_{i}}{\partial x_{i}}+\frac{1}{r} u_{1}=\frac{\partial u_{r}}{\partial r}+\frac{1}{r} \frac{\partial u_{\theta}}{\partial \theta}+\frac{\partial u_{z}}{\partial z}+\frac{u_{r}}{r}\right)$.

These terms do not contribute to the total (integral) turbulent kinetic energy of the whole domain, and only transport the turbulent kinetic from one region to other regions.

Figure 15 shows the turbulent kinetic energy budget at $z=2.5$, and we can see that turbulent energy production term $P$, dissipation term $\varepsilon$, turbulent transport term $T$ and viscous diffusion term $D$ are dominate, and other terms are negligible. The turbulent kinetic energy budget has the following characteristics:

(1) Turbulent energy production term gets the peak near $\eta^{+}=11$, this is the transition region between viscous sublayer and log-law region, and the peak of turbulent intensity is also in this region.

(2) In the viscous sublayer $\left(\eta^{+}<5\right)$, turbulent energy production is not significant, while the turbulent energy dissipation is very strong. Turbulent kinetic energy is transported to this region by turbulent transport term $T$ and viscous diffusion term $D$, and is dissipated by the viscous dissipation term $\varepsilon$.

(3) In the very-near-wall region $\left(\eta^{+}<2\right)$, viscous dissipation term and viscous diffusion term are both very strong, and the two terms balance each other.

(4) The convection term $C$ is not significant because of the quasi-parallel flow and slow growth of the boundary layer. Compared to the convection term of flat-plate boundary layer ${ }^{[11]}$, the turbulent energy convection term of sharp-cone boundary layer seems to be a little larger. This is because that the streamwise variation of the mean flow is a little stronger for the sharp-cone boundary layer than that for the flat-plate boundary layer.

Figure 16 shows the pressure-dilatation term $\Pi_{d}$, pressure transport term $\Pi_{t}$, and the term associated with density fluctuations $M$. These three terms are very small and can be neglected. The reason is that the Mach number is not so high and the intrinsic compressibility is not significant.

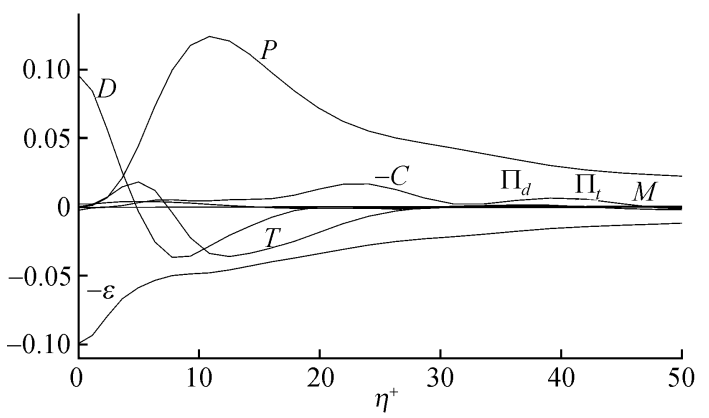

Figure 15 Turbulent kinetic energy budget at $z=2.5$.

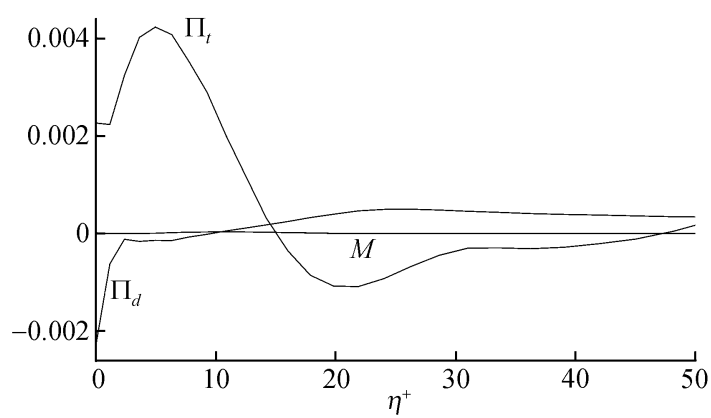

Figure $16 \Pi_{d}, \Pi_{t}$ and $M$ at $z=2.5$.

\section{Conclusions}

In this paper, a direct numerical simulation of turbulent boundary layer around a sharp cone with $20^{\circ}$ cone angle is performed. The free stream Mach number is 0.7 and free stream unit Reynolds number is 250000/inch. Mixed seventh-order up-wind biased difference scheme and sixth-order 
central difference scheme are used in the DNS. Statistics of fully developed turbulent flow agrees well with the experimental and theoretical data for turbulent flat-plate boundary layer flow. Following conclusions can be drawn:

(1) The large amplitude of perturbation and circumferential curvature of cone can change the transition location and reduce the transition region, but it has little effect on turbulent statistics near the wall in the turbulent region such as the mean streamwise velocity, skin friction, turbulent intensity and Reynolds shear stress. This is because the curvature radius of the wall is much greater than the thickness of the boundary layer.

(2) The space between the near wall streaks (normalized by the local wall unit) keeps approximately invariable along $z$-direction.

(3) Hairpin vortex series are found in the transition region.

(4) The product term of turbulent energy reaches its peak in the transition region. Turbulent kinetic energy is transported into the viscous sublayer by turbulent transport term and viscous diffusion term, and is dissipated by the viscous dissipation term. In the very-near-wall region, viscous dissipation term and viscous diffusion term are both very strong, and the two terms balance each other.

\section{Discussion}

The authors think that the transition of this research is bypass-type transition due to the following reasons:

(1) The amplitude of the disturbance is rather strong. In this research, the amplitude of blow-and-suction velocity is $A=0.2$, and this amplitude is much larger than that in the DNS of flat-plate boundary layer ${ }^{[10,11,12]}$.

(2) The wall friction grows very fast just after the disturbance region (see Figure 3), which indicates that the transition begins just after the disturbance region.

\section{Appendix}

\section{A The deduction of the turbulent kinetic energy equation for cylindrical coordinate}

The compressible Navier-Stokes equation for the cylindrical coordinate is as follows:

$$
\begin{gathered}
\frac{\partial \rho}{\partial t}+\frac{\partial\left(\rho u_{i}\right)}{\partial x_{i}}+\frac{1}{r} \rho u_{1}=0 \\
\rho \frac{\partial u_{i}}{\partial t}+\rho u_{j} \frac{\partial u_{i}}{\partial x_{j}}=-\frac{\partial p}{\partial x_{i}}+\frac{\partial \sigma_{i j}}{\partial x_{j}}+s_{i}
\end{gathered}
$$

where

$$
\frac{\partial}{\partial x_{1}}=\frac{\partial}{\partial r}, \frac{\partial}{\partial x_{2}}=\frac{1}{r} \frac{\partial}{\partial \theta}, \frac{\partial}{\partial x_{3}}=\frac{\partial}{\partial z} ; \quad u_{1}=u_{r}, u_{2}=u_{\theta}, u_{3}=u_{z} ;
$$

$s_{i}$ are free terms:

$$
s_{1}=\frac{1}{r}\left(\rho u_{2}^{2}+\sigma_{11}-\sigma_{22}\right), s_{2}=\frac{1}{r}\left(-\rho u_{1} u_{2}+2 \sigma_{12}\right), s_{3}=\frac{1}{r} \sigma_{13} .
$$

Let $\bar{\phi}$ be the Reynolds average of $\phi$, and in this paper, the Reynolds average means the circumference average together with time average. Let $\tilde{\phi}=\overline{\rho \phi} / \bar{\rho}$ be the Favre average of $\phi$. Let 
$\phi^{\prime}=\phi-\bar{\phi}$, and $\phi^{\prime \prime}=\phi-\tilde{\phi}$.

Let $u_{i}^{\prime \prime}$ multiply (A2) and then do some averaging:

$$
\overline{u_{i}^{\prime \prime} \rho \frac{\partial u_{i}}{\partial t}}+\overline{u_{i}^{\prime \prime} \rho_{i} u_{j} \frac{\partial u_{i}}{\partial x_{j}}}=\overline{-u_{i}^{\prime \prime} \frac{\partial p}{\partial x_{i}}}+\overline{u_{i}^{\prime \prime} \frac{\partial}{\partial x_{i}} \sigma_{i j}}+\overline{u_{i}^{\prime \prime} s_{i}} .
$$

Substitute $u_{i}=\tilde{u}_{i}+u_{i}^{\prime \prime}, p=\bar{p}+p^{\prime}, \sigma_{i j}=\bar{\sigma}_{i j}+\sigma_{i j}^{\prime}$ to eq. (A3), and consider eq. (A1):

$$
\begin{aligned}
& \overline{u_{i}^{\prime \prime} \rho \frac{\partial u_{i}}{\partial t}}+\overline{u_{i}^{\prime \prime} \rho u_{j} \frac{\partial u_{i}}{\partial x_{j}}} \\
& =\overline{u_{i}^{\prime \prime} \rho \frac{\partial \tilde{u}_{i}}{\partial t}}+\overline{u_{i}^{\prime \prime} \rho \frac{\partial u_{i}^{\prime \prime}}{\partial t}}+\overline{u_{i}^{\prime \prime} \rho\left(\tilde{u}_{j}+u_{j}^{\prime \prime}\right) \frac{\partial\left(\tilde{u}_{i}+u_{i}^{\prime \prime}\right)}{\partial x_{j}}} \\
& =\overline{1 / 2 \rho \frac{\partial}{\partial t}\left(u_{i}^{\prime \prime} u_{i}^{\prime \prime}\right)}+\overline{u_{j} \rho u_{i}^{\prime \prime} \frac{\partial u_{i}^{\prime \prime}}{\partial x_{j}}}+\overline{u_{i}^{\prime \prime} \rho u_{j}^{\prime \prime} \frac{\partial u_{i}^{\prime \prime}}{\partial x}}+\overline{u_{i}^{\prime \prime} \rho u_{j}^{\prime \prime}} \frac{\partial \tilde{u}_{i}}{\partial x_{j}} \\
& =\overline{1 / 2 \frac{\partial}{\partial t}\left(\rho u_{i}^{\prime \prime} u_{i}^{\prime \prime}\right)}-1 / \overline{2 u_{i}^{\prime \prime} u_{i}^{\prime \prime} \frac{\partial \rho}{\partial t}}+\overline{u_{j} \rho u_{i}^{\prime \prime} \frac{\partial u_{i}^{\prime \prime}}{\partial x_{j}}}+\overline{u_{i}^{\prime \prime} \rho u_{j}^{\prime \prime} \frac{\partial u_{i}^{\prime \prime}}{\partial x}}+\overline{u_{i}^{\prime \prime} \rho u_{j}^{\prime \prime}} \frac{\partial \tilde{u}_{i}}{\partial x_{j}} \\
& =\frac{\partial}{\partial t}(\bar{\rho} K)+\overline{\rho u_{i}^{\prime \prime} u_{j}^{\prime \prime}} \frac{\partial \tilde{u}_{i}}{\partial x_{j}}+1 / 2 \frac{1}{r} \overline{u_{i}^{\prime \prime} u_{i}^{\prime \prime} \rho u_{1}}+1 / \overline{u_{i}^{\prime \prime} u_{i}^{\prime \prime} \frac{\partial \rho u_{j}}{\partial x_{j}}}+\tilde{u}_{j} \overline{\rho u_{i}^{\prime \prime} \frac{\partial u_{i}^{\prime \prime}}{\partial x_{j}}}+\overline{u_{i}^{\prime \prime} \rho u_{j}^{\prime \prime} \frac{\partial u_{i}^{\prime \prime}}{\partial x_{j}}} \\
& =\frac{\partial}{\partial t}(\bar{\rho} K)+\overline{\rho u_{i}^{\prime \prime} u_{j}^{\prime \prime}} \frac{\partial \tilde{u}_{i}}{\partial x_{j}}+1 / \overline{2 u_{i}^{\prime \prime} u_{i}^{\prime \prime} \frac{\partial \rho u_{j}^{\prime \prime}}{\partial x_{j}}}+1 / 2 \overline{u_{i}^{\prime \prime} u_{i}^{\prime \prime} \frac{\partial \rho \tilde{u}_{j}}{\partial x_{j}}}+1 / 2 \rho \tilde{u}_{j} \frac{\partial u_{i}^{\prime \prime} u_{i}^{\prime \prime}}{\partial x_{j}} \\
& +1 / 2 \rho u_{j}^{\prime \prime} \frac{\partial u_{i}^{\prime \prime} u_{i}^{\prime \prime}}{\partial x_{j}}+1 / 2 \frac{1}{r} \overline{u_{i}^{\prime \prime} u_{i}^{\prime \prime} \rho u_{1}} \\
& =\frac{\partial}{\partial t}(\bar{\rho} K)+\overline{\rho u_{i}^{\prime \prime} u_{j}^{\prime \prime}} \frac{\partial \tilde{u}_{i}}{\partial x_{j}}+\left(1 / \overline{u_{i}^{\prime \prime} u_{i}^{\prime \prime} \frac{\partial \rho u_{j}^{\prime \prime}}{\partial x_{j}}}+1 / 2 \overline{\rho u_{j}^{\prime \prime} \frac{\partial u_{i}^{\prime \prime} u_{i}^{\prime \prime}}{\partial x_{j}}}\right)+\left(1 / 2 \overline{u_{i}^{\prime \prime} u_{i}^{\prime \prime} \frac{\partial \rho \tilde{u}_{j}}{\partial x_{j}}}+1 / 2 \overline{\rho \tilde{u}_{j} \frac{\partial u_{i}^{\prime \prime} u_{i}^{\prime \prime}}{\partial x_{j}}}\right) \\
& +1 / 2 \frac{1}{r} \overline{u_{i}^{\prime \prime} u_{i}^{\prime \prime} \rho u_{1}^{\prime \prime}}+1 / 2 \frac{1}{r} \overline{u_{i}^{\prime \prime} u_{i}^{\prime \prime} \rho \tilde{u}_{1}} \\
& =\frac{\partial}{\partial t}(\bar{\rho} K)+\overline{\rho u_{i}^{\prime \prime} u_{j}^{\prime \prime}} \frac{\partial \tilde{u}_{i}}{\partial x_{j}}+1 / 2 \frac{1}{r} \overline{u_{i}^{\prime \prime} u_{i}^{\prime \prime} \rho u_{1}}+1 / 2 \frac{\partial}{\partial x_{j}} \overline{\rho u_{i}^{\prime \prime} u_{i}^{\prime \prime} u_{j}^{\prime \prime}}+\frac{\partial}{\partial x_{j}}\left(\tilde{u}_{j} \bar{\rho} K\right) \\
& +\frac{1}{r} \bar{\rho} K \tilde{u}_{1}+1 / 2 \frac{1}{r} \overline{\rho u_{i}^{\prime \prime} u_{i}^{\prime \prime} u_{1}^{\prime \prime}},
\end{aligned}
$$

where $K=1 / 2 \overline{\rho u_{i}^{\prime \prime} u_{i}^{\prime \prime}} / \bar{\rho}$ is turbulent kinetic energy.

Considering the other part of turbulent kinetic energy equation, the first two terms on the right hand side of eq. (A3) are

$$
\begin{aligned}
& \overline{-u_{i}^{\prime \prime} \frac{\partial p}{\partial x_{i}}}+\overline{u_{i}^{\prime \prime} \frac{\partial}{\partial x_{j}} \sigma_{i j}}=\overline{-u_{i}^{\prime \prime} \frac{\partial\left(\bar{p}+p^{\prime}\right)}{\partial x_{i}}}+\overline{u_{i}^{\prime \prime} \frac{\partial\left(\bar{\sigma}_{i j}+\sigma_{i j}^{\prime}\right)}{\partial x_{j}}} \\
& =\vec{u}_{i}^{\prime \prime}\left(\frac{\partial \bar{\sigma}_{i j}}{\partial x_{j}}-\frac{\partial \bar{p}}{\partial x_{i}}\right)-\frac{\partial}{\partial x_{i}}\left(\overline{u_{i}^{\prime \prime} p^{\prime}}\right)+\overline{p^{\prime} \frac{\partial u_{i}^{\prime \prime}}{\partial x_{i}}}+\frac{\partial}{\partial x_{i}} \overline{u_{i}^{\prime \prime} \sigma_{i j}^{\prime}}-\overline{\sigma_{i j}^{\prime} \frac{\partial u_{i}^{\prime \prime}}{\partial x_{j}}} .
\end{aligned}
$$

The last term of eq. (A3) is 


$$
\begin{aligned}
\overline{u_{i}^{\prime \prime} s_{i}}= & \frac{1}{r} \overline{\rho\left(u_{2}^{2} u_{1}^{\prime \prime}-u_{1} u_{2} u_{2}^{\prime \prime}\right)}+\frac{1}{r} \overline{\left(\sigma_{11}-\sigma_{12}\right) u_{1}^{\prime \prime}+2 \sigma_{12} u_{2}^{\prime \prime}+\sigma_{13} u_{3}^{\prime \prime}} \\
= & \frac{1}{r}\left(\tilde{u}_{2} \overline{\rho u_{1}^{\prime \prime} u_{2}^{\prime \prime}}-\tilde{u}_{1} \overline{\rho u_{2}^{\prime \prime} u_{2}^{\prime \prime}}\right)+\frac{1}{r}\left(\overline{u_{1}^{\prime \prime}}\left(\bar{\sigma}_{11}-\bar{\sigma}_{12}\right)+2 \overline{u_{2}^{\prime \prime}} \bar{\sigma}_{12}+\overline{u_{3}^{\prime \prime}} \bar{\sigma}_{13}\right) \\
& +\frac{1}{r} \overline{\left(\sigma_{11}^{\prime}-\sigma_{12}^{\prime}\right) u_{1}^{\prime \prime}+2 \sigma_{12}^{\prime} u_{2}^{\prime \prime}+\sigma_{13}^{\prime} u_{3}^{\prime \prime} .}
\end{aligned}
$$

Substituting eqs. (A4)-(A6) to (A3), we get the turbulent kinetic energy equation for the cylindrical coordinate:

$$
\frac{\partial}{\partial t}(\bar{\rho} K)=-C+P+T+\Pi_{t}+\Pi_{d}+D+M-\varepsilon
$$

where $C=\frac{\partial}{\partial x_{j}}\left(\tilde{u}_{j} \bar{\rho} K\right)+\frac{1}{r} \tilde{u}_{1} \bar{\rho} K$ is the convection term;

$$
P=-\overline{\rho u_{i}^{\prime \prime} u_{j}^{\prime \prime}} \frac{\partial \tilde{u}_{i}}{\partial x_{j}}+\frac{1}{r}\left(\tilde{u}_{2} \overline{\rho u_{1}^{\prime \prime} u_{2}^{\prime \prime}}-\tilde{u}_{1} \overline{\rho u_{2}^{\prime \prime} u_{2}^{\prime \prime}}\right)
$$

is the production term;

$$
T=-\left(\frac{\partial}{\partial x_{j}}\left(1 / 2 \overline{\rho u_{i}^{\prime \prime} u_{i}^{\prime \prime} u_{j}^{\prime \prime}}\right)+1 / 2 \frac{1}{r} \overline{\rho u_{i}^{\prime \prime} u_{i}^{\prime \prime} u_{1}^{\prime \prime}}\right)
$$

is the turbulent transport term;

$$
\Pi_{d}=\overline{p^{\prime}\left(\frac{\partial u_{i}^{\prime \prime}}{\partial x_{i}}+\frac{1}{r} u_{1}^{\prime \prime}\right)}
$$

is the pressure-dilatation term;

$$
\Pi_{t}=-\left(\frac{\partial}{\partial x_{j}} \overline{p^{\prime} u_{j}^{\prime \prime}}+\frac{1}{r} \overline{p^{\prime} u_{1}^{\prime \prime}}\right)
$$

is the pressure transport term;

$$
M=\bar{u}_{i}^{\prime \prime}\left(\frac{\partial \bar{\sigma}_{i j}}{\partial x_{j}}-\frac{\partial \bar{p}}{\partial x_{i}}\right)+\frac{1}{r}\left(\overline{u_{1}^{\prime \prime}}\left(\bar{\sigma}_{11}-\bar{\sigma}_{12}\right)+2 \overline{u_{2}^{\prime \prime}} \bar{\sigma}_{12}+\overline{u_{3}^{\prime \prime}} \bar{\sigma}_{13}\right)
$$

is the term associated with density fluctuations;

$$
D=\frac{\partial}{\partial x_{i}} \overline{u_{i}^{\prime \prime} \sigma_{i j}^{\prime}}+\frac{1}{r} \overline{u_{i}^{\prime \prime} \sigma_{i 1}^{\prime}}
$$

is the viscous diffusion term;

$$
\varepsilon=\overline{\sigma_{i j}^{\prime} \frac{\partial u_{i}^{\prime \prime}}{\partial x_{j}}}+\frac{1}{r} \overline{\sigma_{12}^{\prime} u_{2}^{\prime \prime}-\sigma_{12}^{\prime} u_{1}^{\prime \prime}}
$$

is the viscous dissipation term.

The authors would like to thank Shanghai Supercomputer Center (SSC) and Supercomputing Center of Chinese Academy of Sciences (SCCAS) for providing computer time. Thanks go to Profs. Zhou Heng, Luo Jiseng and Cao Wei of Tianjin University for helpful discussion.

1 Schneider S P. Flight data for boundary-layer transition at hypersonic and supersonic speeds. J Spacecra Rockets, 1998, 36(1): $8-20$

2 Rabb L, Simpkinson H. Free flight heat transfer measurements on two $20^{\circ}$ cone cylinders at Mach number from 1.3 to 4.9 . 
NACA Res Memo, 1955, NACA-RM-E55F27

3 Rabb L, Disher J H. Boundary layer transition at high Reynolds numbers as obtained in flight of a $20^{\circ}$ cone-cylinder with wall to local stream temperature ratios near 1.0. NACA Res Memo, 1955, NACA-RM-E55I15

4 Chauvin L T, Speegle K C. Boundary layer transition and heat transfer measurements for flight tests of blunt and sharp $50^{\circ}$ cones at Mach numbers from 1.7 to 4.7. NACA Res Memo, 1957, NACA-RM-L57D04

5 Ma Y B, Zhong X L. Receptivity of a supersonic boundary layer over a flat plate (I): Wave structures and interactions, J Fluid Mech, 2003, 488: 31-78

6 Zhong X L, Ma Y B. Boundary-layer receptivity of Mach 7.99 flow over a blunt cone to free-stream acoustic waves. J Fluid Mech, 2006, 556: 53-103

7 Zhong X L. Numerical simulation of surface roughness effects on receptivity of hypersonic flow over blunt cones. AIAA Paper, 2007: 2007-0944

8 Guarini S E, Moser R D, Shariff K, et al. Direct numerical simulation of a supersonic turbulent boundary layer at Mach 2.5. J Fluid Mech, 2000, 414: 1-33

9 Maeder T, Adams N A, Kleiser L. Direct simulation of turbulent supersonic boundary layer by an extended temporal approach. J Fluid Mech, 2001, 429: 187-216[DOI]

10 Rai M M, Gatski T B, Erlebacher G. Direct numerical simulation of spatially evolving compressible turbulent boundary layers. AIAA paper, 1995: 95-0583

11 Pirozzoli S, Grasso F. Direct numerical simulation and analysis of a spatially evolving supersonic turbulent boundary layer at $M=2.25$. Phys Fluids, 2004, 16(3): 530-545[DOI]

12 Gao H, Fu D X, Ma Y W, et al. Direct numerical simulation of supersonic boundary layer flow. Chin Phys Lett, 2005, 22(7): $1709-1712$ [DOI]

13 Luo J S, Wang X J, Zhou H. Inherent mechanism of breakdown in laminar-turbulent transition of plane channel flows. Sci China Ser G-Phys Mech Astron, 2005, 48(2): 228-236

14 Li X L, Fu D X, Ma Y W. DNS of compressible turbulent boundary layer over a blunt wedge. Sci China Ser G-Phys Mech Astron, 2005, 48(2): 129-141

15 Li X L, Fu D X, Ma Y W, Direct numerical simulation of a spatially evolving supersonic turbulent boundary layer at Ma= 6. Chin Phys Lett, 2006, 23(6): 1519-1522[DOI]

16 Horvath T J, Berry S A, Hollis B R, et al. Boundary layer transition on slender cones in conventional and low disturbance Mach 6 wind tunnels. AIAA Paper, 2002: 2002-2743

17 Fu D X, Ma Y W. A high order accurate different scheme for complex flow fields. Comput Phys, 1997, 134: 1 - 15[DOI]

18 Fu D X, Ma Y W. High resolution schemes. In: Hafez M, Oshima K, eds. Computational Fluid Dynamics Review. New York: John Wiley \& Sons, 1995. 234-250

19 Li X L, Fu D X, Ma Y W. Optimized group velocity control scheme and DNS of decaying compressible turbulence of relative high turbulent Mach number. Int Numer Mech Fluids, 48, 2005: 835 - 852[DOI]

20 Karlson R I, Johansson T G. LDV measurements of higher-order moments of velocity fluctuations in a turbulent boundary layer. In: Laser Anemometry in Fluid Mechanics. Lisbon Portugal: Ladoan-Instituto Superior Tecnico, 1986 